\title{
Electro-Mechanical Delay in the Human Heart: A Study on a Simple Geometry
}

\author{
Ekaterina Kovacheva', Lukas Baron', Olaf Dössel', Axel Loewe' \\ 'Institute of Biomedical Engineering, Karlsruhe Institute of Technology (KIT) \\ Karlsruhe, Germany
}

\begin{abstract}
The contraction of the heart is a complex process involving the interaction of the passive properties of the tissue and the active tension development, which is elicited by the electrical activation of the cells.

In this study, the electro-mechanical delay (EMD) was investigated as well as its dependence on the length of the sarcomeres, which are the contractile units within the cell. $E M D$ was defined as the time offset between the electrical activation of the cell and the time of maximal tension.

On a simple bar geometry with unidirectional fibre orientation and a linear local activation time distribution, the EMD proved to be inhomogeneous. The contraction of the early activated regions caused an elongation of the sarcomere (stretch) in the neighbouring regions, which ware electrically activated at a later time. The tension in the stretched region reached twice the value of the cells in the not-stretched, early activated region. Furthermore, the $E M D$ in the early electrically activated region was more than $0.2 \mathrm{~s}$, which was about twice the EMD of the stretched regions.

In conclusion, the stretched region developed higher tension within a shorter time interval compared to the early activated region. Future studies will investigate how the inhomogeneous EMD affects cardiac output.
\end{abstract}

\section{Introduction}

During the heart cycle, the myocytes are activated electrically, which is reflected in the action potential. A large fraction of the intracellular volume is filled with sarcomeres, which develop tension upon electrical excitation. This causes the shortening of each myocyte and altogether the mechanical contraction of the heart.

The electro-mechanical delay (EMD) is the offset between the electrical activation and mechanical activation, which is related to the tension development by the myocyte. The course of the tension development, depends on the stretch of the sarcomere causing the EMD to be related to the stretch upon excitation. EMD and in particular its heterogeneity might play crucial roles in achieving hemodynamic efficiency and could be involved in arrhythmogenesis.

\section{Methods}

\subsection{Geometry Model}

To focus on the fundamental mechanisms affecting EMD, this study is based on a simple geometry. We considered a beam geometry of dimension $100 \mathrm{~mm} \times$ $15 \mathrm{~mm} \times 15 \mathrm{~mm}$. It represents an apico-basal section of the left ventricle as shown in Figure 1.

The beam was discretized by 961 quadratic tetrahedrons comprising 1840 nodes. The lower, blue part represents the myocardium (active beam). The end of the active beam, which represents the base of the heart, a further beam (passive beam) was attached simulating a spring. The passive beam was fixed at the top and damped the contraction of the active beam limited atrioventricular

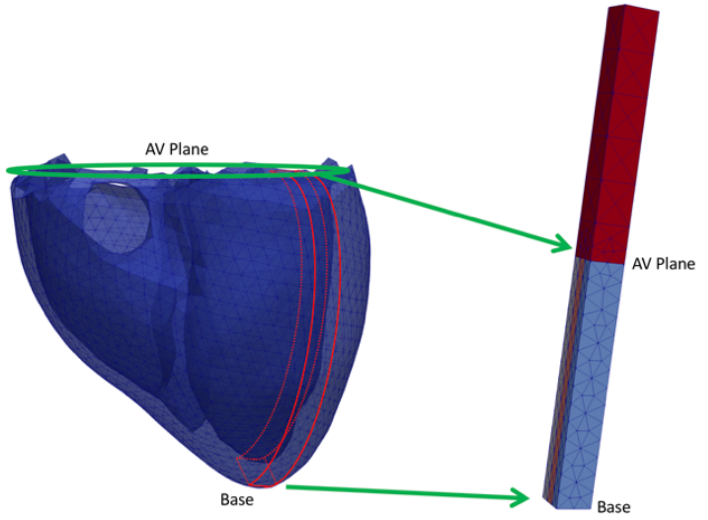

Figure 1: Beam geometry representing a part of the left ventricle. 


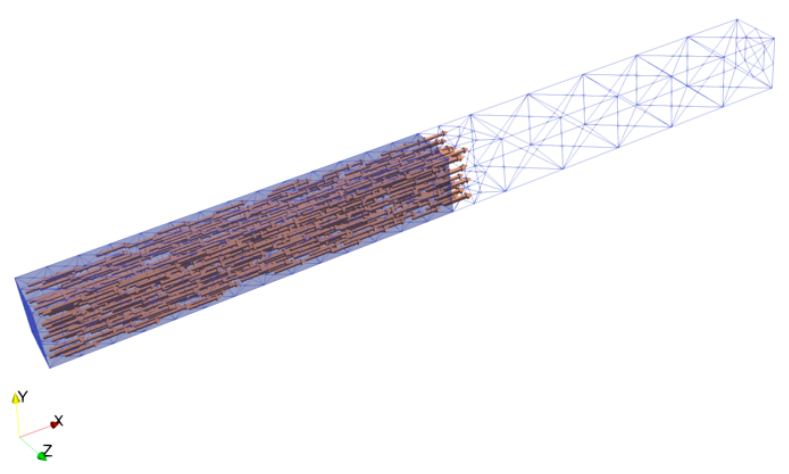

Figure 2: Fibers of the active beam.

(AV) plane movement to around $17 \mathrm{~mm}$. Furthermore, it controls the stretch experienced by the cells in the active beam. The active beam was fixed on the apical side. The fibers of the active beam were modeled unidirectional along the main axis as visualized in Figure 2.

The contraction of the beam was simulated by the finite element method as implemented in the work of Fritz [4].

Although the fibers of the human heart are aligned in circumferential direction with a varying angle from the epicardial to the endocardial surface, the simple beam geometry required a simplified fiber setup.

\subsection{Passive and Active Material Properties}

The passive properties of the active beam were described by the transversely isotropic Guccione material law [1], which was formulated for ventricular tissue. The passive properties of the passive beam were described by the nonlinear hyperelastic Mooney-Rivlin constitutive model, which was designed to reflect the atrial material properties. Model parameters are provided in Table 1.

\begin{tabular}{|c|cccc|ccc|}
\hline & \multicolumn{4}{|c|}{ Guccione } & \multicolumn{3}{c|}{ Mooney Rivlin } \\
\hline C & b1 & b2 & b3 & K & C1 & C2 & B \\
\hline 60 & 26.7 & 2.0 & 14.7 & $2 \mathrm{e} 5$ & 7450 & 0 & $2 \mathrm{e} 5$ \\
\hline
\end{tabular}

Table 1. Guccione and Mooney-Rivlin model parameters.

The active properties, i.e., the tension development, of the active beam were modelled using the Lumens model [2]. It generates the tension in fibre direction depending on the stretch and using a fixed calcium concentration curve, which is activated by the local activation time.

\subsection{Local Activation Time}

The local activation time (LAT) was modelled with a linear distribution along the active beam. LAT was zero at the apical end and increased linearly to $0.1 \mathrm{~s}$ at the basal end representing the AV plane (Figure 3).

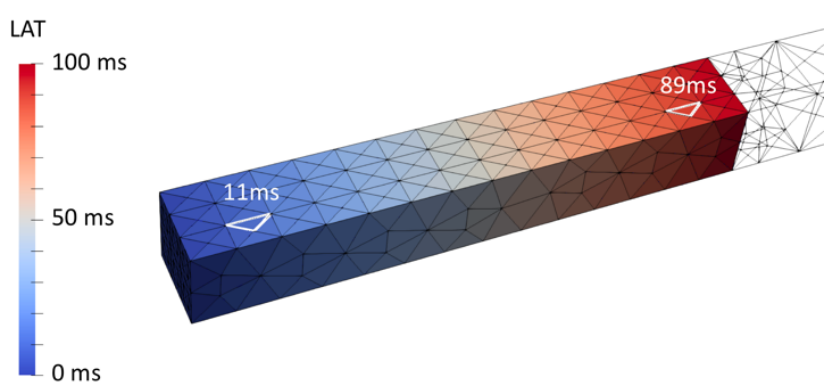

Figure 3: Linear LAT map of the active beam.

\section{Results}

We evaluated the EMD along the beam as well as its dependence on the stretch of the cells. In the following the tension and the stretch are visualized for two elements and for all element along a line parallel to the long axis.

\subsection{Tension and Stretch}

In Figure 3, two elements are marked by the white color and their corresponding activation time: the early activated one is in the region near the fixed end, which represents the apex and late activated element is in the region near the end which represents the AV plane and is attached to the passive beam (partly visualized as a surface mesh).

For both elements the temporal tension development are shown in Figure 4A: the blue curve describes the tension developed by the early activated element and the red curve - the tension developed by the late activated element. In Figure $4 \mathrm{~B}$ the stretch values bigger than 1 correspond to elongation of the sarcomere, whereas values smaller than 1 correspond to shortening.

The element, which is electrically activated $11 \mathrm{~ms}$ after
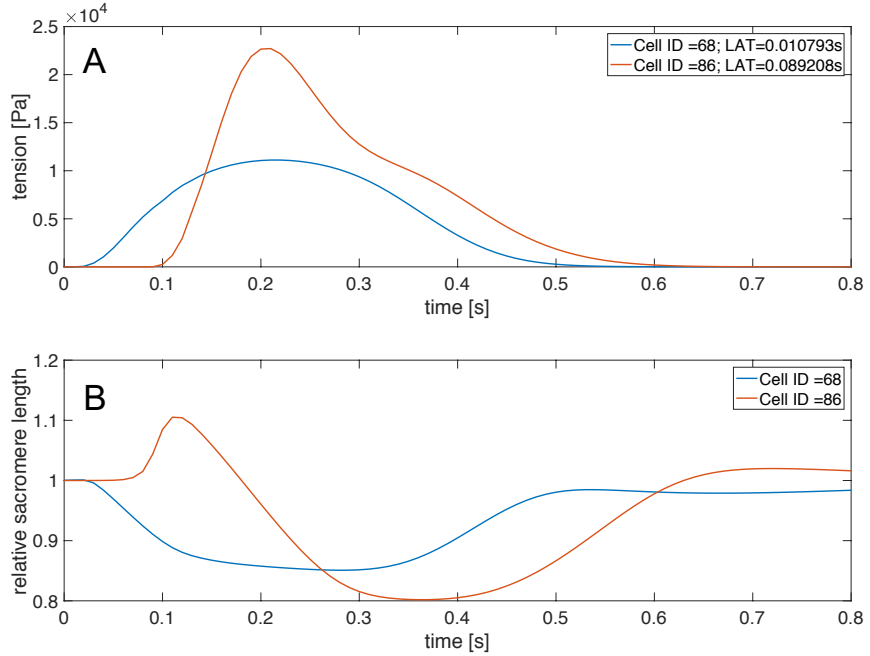

Figure 4: (A) Tension development and (B) stretch over time for an early activated element (blue curves) and late activated element (red curves). 
simulation start belongs to the early activated region and thus, does not experience any stretch. The developed tension reaches a value of about $10 \mathrm{kPa}$ and the sarcomere contracts to about $85 \%$ of its initial length. The shortening of the cells in the early activated region causes a stretch in its neighboring region. This can be observed, for instance, for the element, which is electrically activated $89 \mathrm{~ms}$ after simulation start. In Figure 4B, it is apparent that the element is firstly elongated to about $110 \%$ of initial length and then contracts to reach about $80 \%$ of the initial length. The shortening of the initially stretched elements happens faster than in the early activated elements. Furthermore, the developed tension reaches values of about $22 \mathrm{kPa}$, again more rapidly, as visualized in Figure 4A. The developed tension in the stretched regions is more than double the value of early activated regions.

For a more global view, 15 elements along the main axis of the beam were observed. Tension and stretch curves showed a continuous progression from the early to the late activated elements (Figure 5).

While observing the elements consecutively from the apex to the base end, the maximum developed tension reaches higher values and the stretch raises evenly. Furthermore, the tension develops quicker and the resulting contraction leads to shorter cell length.

This can be also observed in the plots in Figure 6, where the $\mathrm{x}$ axis depicts the element id along the line and the $\mathrm{y}$ axis corresponds to the maximum developed tension, which gradually increases. Analogically, in Figure 7A and $\mathrm{B}$ the stretch at activation time and the maximum relative length of the cell increases, while the minimum stretch decreases as visualized in Figure 7C.
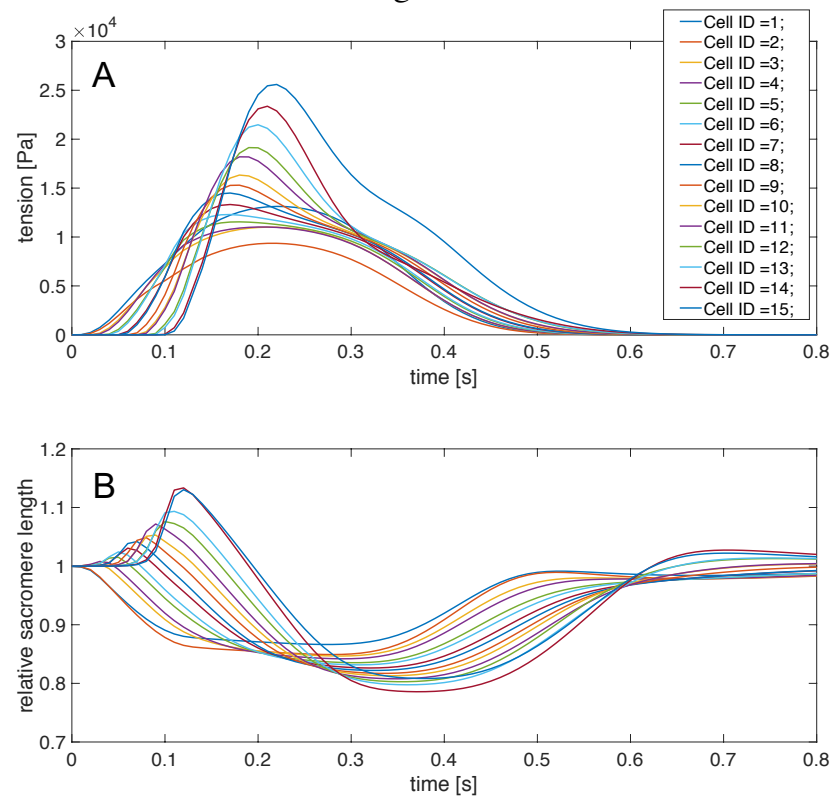

Figure 5: (A) Tension development and (B) stretch of 15 elements aligned in a line. The element with id $=1$ is the first activated one, while the element with id $=15$ is the last activated element.

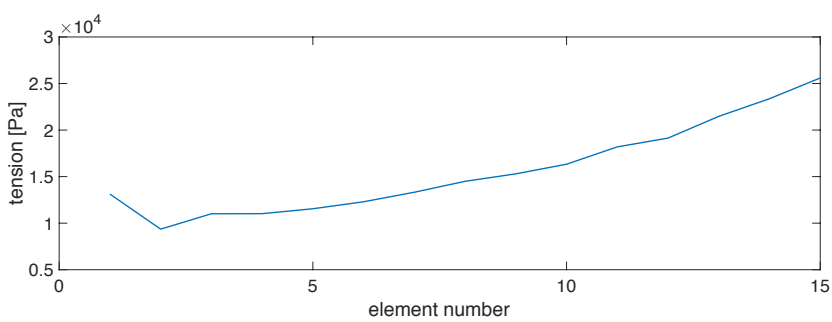

Figure 6: The maximum developed tension visualized for each element on the observed line.

\subsection{Electro-Mechanical Delay}

The EMD for one cell is calculated as the time offset between the electrical activation and the mechanical activation, which is the time of maximal tension. Alternatively, one could define the mechanical activation as the time point of maximal contraction (minimal stretch) or the time point when the shortening reaches a certain percentage of its maximum as done by Gurev and Trayanova [3]. The spatial EMD distribution is visualized in Figure 8.
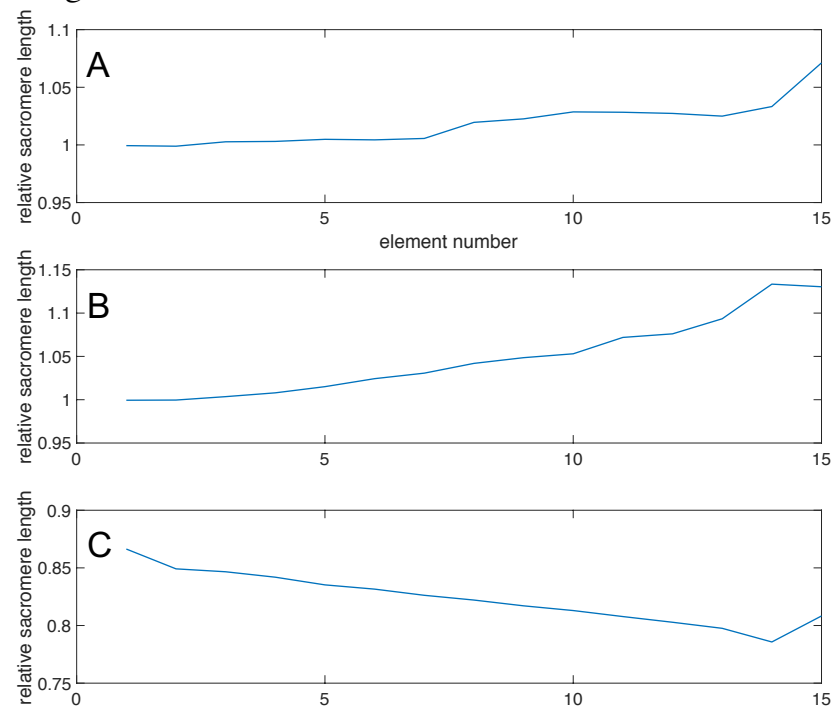

Figure 7: The stretch is visualized for each element: (A) at activation time, (B) the maximum stretch and (C) the minimum stretch. 


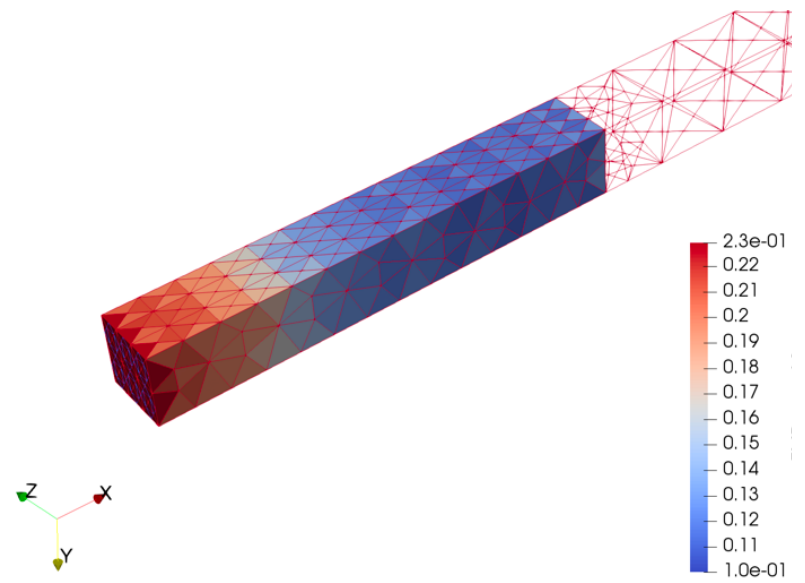

Figure 8: EMD distribution.

The maximal value of the EMD is $0.22 \mathrm{~s}$ which was observed in the early activated region. The minimal value of the EMD is $0.10 \mathrm{~s}$ and was observed in the stretched regions as the tension develops quicker compared to the early activated regions.

In Figure 9, one can observe the EMD for the elements aligned over the line, as described previously. The EMD decreased continuously from the apex to the base.

\section{Conclusion}

This study evaluated the EMD and its dependence on the stretch caused by other adjacent regions. The simple beam geometry was used in order to exclude effects arising from a complex geometry. A linear LAT map was predefined to electrically activate the cells in a consecutive manner starting from the end representing the apex and reaching its maximum at the end representing the base. The beam was subdivided in two regions with different behavior. The region of the early activated elements did not undergo any stretch. It contracted while the tension was developed. As a result, the neighboring region was stretched and developed higher tension in shorter time. The length of the contracted elements was also shorter. Thus, the stretched regions turned out to have a shorter EMD compared to not stretched ones.

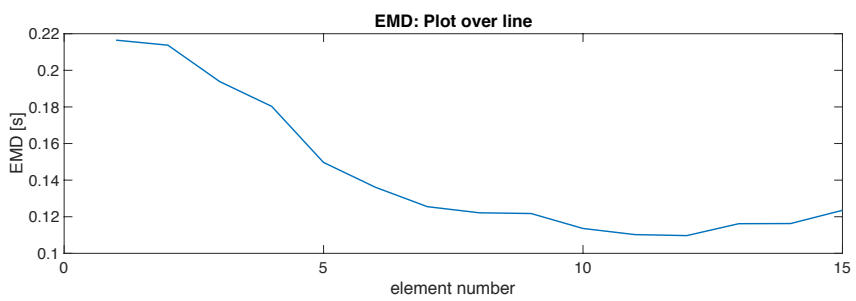

Figure 9: EMD visualized for the elements aligned over a line.

\section{References}

[1] Omens. Measurement of Strain and Analysis of Stress in Resting Rat Left Ventricular Myocardium. In: Journal Biomechanics, No. 6, 1993; 26:665-676.

[2] Lumens. Three-Wall Segment (TriSeg) Model Describing Mechanics and Hemodynamics of Ventricular Interaction. In: Annals of Biomedical Engineering, No. 11, November 2009;37:2234-2255.

[3] Gurev, Trayanova. Distribution of Electromechanical Delay in the Heart: Insights from a Three-Dimensional Electromechanical Model. In: Biophysical Journal, 2010; 99:745-754.

[4] Simulation of the Contraction of the Ventricles in a Human Heart Model Including Atria and Pericardium: Finite Element Analysis of a Frictionless Contact Problem. In: Biomech Model Mechanobiol (2014);13:627-641.

We gratefully acknowledge financial support by the Deutsche Forschungsgemeinschaft (DFG) through CRC 1173.

Ekaterina Kovacheva

Institute of Biomedical Engineering, Karlsruhe Institute of Technology (KIT), Kaiserstr. 12, 76131, Karlsruhe, Germany. publications@ibt.kit.edu 